\title{
POTENCIALIDADES DA TÉCNICA DE GRUPO FOCAL PARA A PESQUISA EM VIGILÂNCIA SANITÁRIA E ATENÇÃO PRIMÁRIA À SAÚDE
}

\section{POTENTIAL OF THE FOCUS GROUP TECHNIQUE FOR RESEARCH IN HEALTH SURVEILLANCE AND PRIMARY HEALTH CARE}

\author{
Mariluce Karla Bomfim de Souza ${ }^{1}$ \\ Yara Oyram Ramos Lima ${ }^{2}$ \\ Bianca Maria Santos da Paz ${ }^{3}$ \\ Ediná Alves Costa ${ }^{4}$ \\ Alcione Brasileiro Oliveira Cunha ${ }^{5}$ \\ Rosenilde dos Santos ${ }^{6}$
}

\begin{abstract}
Resumo: Este artigo teve por objetivo discutir potencialidades do grupo focal (GF) para o desenvolvimento de pesquisa sobre Vigilância Sanitária e Atenção Primária à Saúde. Foram realizados GF: com técnicos da vigilância sanitária municipal e profissionais das equipes de saúde da família. Para planejamento e montagem, foram observadas questões orçamentárias e estruturais, organizados roteiros, estabelecidas responsabilidades e selecionados os participantes. Os GF transcorreram com duplas de moderadores e observadores, suporte tecnológico e respeito aos aspectos éticos. Desafios não suplantaram potencialidades do uso da técnica, como a possibilidade de vocalização de profissionais da Vigilância Sanitária pela especificidade das atividades regulatórias, a neutralidade do ambiente para a realização da discussão entre os participantes, a oportunidade de diálogo gerada pela interação e a possibilidade de revisitar a realidade pelo olhar do outro. Conclui-se que a aplicação de uma técnica de pesquisa com rigor metodológico é condição necessária para a produção científica de qualidade.
\end{abstract}

Palavras chave: Grupos Focais; Vigilância Sanitária; Atenção Primária à Saúde.

Abstract: This article aimed to highlight the potential of the focus group (FG) for the development of research on Health Surveillance and Primary Health Care. GF were performed: with the municipal health sanitation and the professionals of the family health teams. To the planning and setting, in which not allowed, and the organized rotaries, greens and the tasks the storages. The FGs had double sessions of moderators and observers, technological support and respect for ethical aspects. Challenges did not supplant the potentialities of the use of the technique, as a possibility of vocalization of health professionals. The discipline of regulatory activities, a neutrality of the environment for an accomplishment of the task among

\footnotetext{
${ }^{1}$ Doutora em Saúde Pública pela Universidade Federal da Bahia (UFBA). Professora do Instituto de Saúde Coletiva da Universidade Federal da Bahia (ISC/UFBA), Salvador, Bahia, Brasil. E-mail: marilucejbv@yahoo.com.br

${ }^{2}$ Doutora em Saúde Pública, Universidade Federal da Bahia (UFBA). Professora do Instituto de Saúde Coletiva da Universidade Federal da Bahia (ISC/UFBA), Salvador, Bahia, Brasil. E-mail: oyram@ufba.br ${ }^{3}$ Mestre em Saúde Coletiva/Vigilância Sanitária, Universidade Federal da Bahia (UFBA). Nutricionista Pesquisadora do Instituto de Saúde Coletiva da Universidade Federal da Bahia (ISC/UFBA), Salvador, Bahia, Brasil. E-mail: bpaz@ufba.br

${ }^{4}$ Doutora em Saúde Pública, Universidade de São Paulo (USP). Professora do Instituto de Saúde Coletiva da Universidade Federal da Bahia (ISC/UFBA), Salvador, Bahia, Brasil. E-mail: edina@ufba.br

${ }^{5}$ Doutora em Saúde Pública, Universidade Federal da Bahia (UFBA). Professora do Instituto de Saúde Coletiva da Universidade Federal da Bahia (ISC/UFBA), Salvador, Bahia, Brasil. E-mail: acunha@ ufba.br ${ }^{6}$ Mestre em Saúde Coletiva/Vigilância Sanitária pela Universidade Federal da Bahia (UFBA), Salvador, Bahia. E-mail: rosedsantos@yahoo.com.br
} 
the participants, an opportunity for dialogue generated by the interaction and a possibility to revisit a research by the method of a rigorous research technique is necessary for a scientific production of quality.

Keywords: Focus Groups; Health Surveillance; Primary Health Care.

\section{Introdução}

Ao longo das últimas décadas tem-se ampliado o interesse pelo estudo dos sistemas e serviços de saúde e suas relações com a saúde das populações. No Brasil, o tema das condições sanitárias dos serviços de saúde vem crescendo em importância com a ocorrência de eventos adversos de impacto na saúde da população. A tragédia radioativa de Goiânia nos anos 1980, a da clínica de hemodiálise em Caruaru-Pernambuco e das mortes de bebês em Unidades de Terapia Intensiva Neonatais nos anos 1990, entre outras, demarcaram um novo estágio na percepção dos riscos inerentes às tecnologias em uso nos serviços de saúde e a necessidade de ações regulatórias efetivas (COSTA, SOUTO, 2013), despertando o interesse de pesquisadores, trabalhadores e gestores da saúde.

Estas preocupações motivaram a realização de um estudo no âmbito da Atenção Primária à Saúde (APS), em uma capital do Nordeste brasileiro, recortando-se as Unidades de Saúde da Família (USF), com o objetivo de analisar as condições sanitárias das USF e como estas condições poderiam interferir no processo de trabalho das equipes, na saúde da população e na gestão (SOUZA et al., 2015). A metodologia do estudo constou de observação das USF selecionadas, mediante um roteiro estruturado com base nos requisitos para o funcionamento das unidades de saúde constantes das normativas de atenção primária à saúde e de vigilância sanitária, além da realização de entrevistas individuais com usuários e gestores e da técnica de grupo focal (GF), objeto deste artigo.

O GF se constitui em uma técnica fundamentada na discussão e interação de ideias, consubstanciando a construção de conhecimentos e a intersubjetividade (THOFEHRN et al., 2013; DIAS, 2000). São grupos de discussão que dialogam sobre um tema ao receberem estímulos apropriados para o debate, conformando, portanto, uma técnica de coleta de dados adequada para pesquisas qualitativas que tem como vantagens ampliar a compreensão sobre um tema específico, favorecer descobertas e trocas de informações culturalmente intrínsecas, além de oportunizar a interpretação de crenças, valores, pontos de vista, conflitos, conceitos etc., ou seja, tem um cunho exploratório (RESSEL et al., 2008), podendo ser usada como técnica complementar em pesquisas quantitativas (MERTON; FISKE; KENDALL, 1990). 
A literatura apresenta versões e datas diversificadas para a origem dessa técnica e refere que a mesma foi descrita e publicada desde 1926, em pesquisas na área das Ciências Sociais, utilizando-se entrevistas grupais (RESSEL et al., 2008). De acordo com Costa (2012), o pai desta técnica foi Merton e teria surgido como uma estratégia para avaliar reações às propagandas e transmissão de rádio durante a Segunda Guerra Mundial. A partir dos anos 80 essa técnica passou a ser aperfeiçoada e despertou um interesse maior para as áreas de marketing e saúde (THOFEHRN et al., 2013).

Nos dias atuais os GF vêm sendo mais utilizados, o que pode ser atribuído à simplicidade da execução e rapidez com que se obtém o mesmo volume de dados provenientes de informantes diversos quando comparados com outros métodos, além do seu baixo custo relativo, a exemplo da entrevista individual, despertando assim o interesse na utilização desta técnica em muitos estudos ao redor do mundo e no Brasil (TRAD, 2009).

No âmbito da saúde, o GF vem sendo muito empregado em temas relacionados à enfermagem e à saúde da família. Neste último caso, essencialmente para avaliação do Programa Saúde da Família e para avaliar a necessidade de sua implantação em uma comunidade. As principais indicações para a opção por esta técnica são os temas pouco estudados, pois os GF permitem a investigação da percepção dos anseios de um determinado grupo sobre temáticas de exploração inicial, o que possibilita a utilização de outras metodologias para abordagem do tema (IERVOLINO; PELICIONI, 2001).

Um pré-requisito básico para a montagem de um GF é a elaboração de um projeto de investigação com objetivos bem definidos (SOUZA et al., 2015), não sendo recomendado seu uso quando o assunto for de cunho constrangedor e o pesquisador não puder controlar aspectos críticos sobre o tema investigado (SCHROEDER; KLERING, 2009). Podem-se considerar desafios para o uso da técnica de GF a exigência por entrevistadores treinados, as possíveis dificuldades para reunir o grupo e manter o clima de diálogo entre os participantes e a execução do procedimento de análise dos dados. Entretanto, se constituem como vantagens e facilidades, a possibilidade de ampliação da amostra, a alta validade dos dados coletados e a habilidade de explorar tópicos e gerar hipóteses (KRUEGER, 1994).

A escolha da técnica de GF para o desenvolvimento da pesquisa em Vigilância Sanitária e Atenção Primária à Saúde se deu pela oportunidade de reunir em cada encontro pessoas/trabalhadores que operam com objetos, meios e finalidades de trabalho afins, 
possibilitando, portanto, a discussão, reflexão e análise crítica sobre as condições sanitárias das USF com maior liberdade e autonomia para os relatos e depoimentos.

A vigilância sanitária, em que pese constituir, em suas origens, a configuração mais antiga da Saúde Pública e atualmente representar sua face mais complexa (COSTA; ROZENFELD, 2000), só mais recentemente surgiu, no Brasil, como tema emergente na pesquisa e na formação de pessoal em saúde. Embora se observe um crescimento da produção científica no tema, permanecem muitas lacunas, inclusive acerca das percepções dos trabalhadores sobre sua atuação nos serviços de saúde e dos profissionais de saúde e gestores dos serviços sobre o exercício da vigilância sanitária (COSTA; SOUTO, 2013). Neste sentido, questionam-se as potencialidades da técnica de GF para a identificação e a discussão sobre os instrumentos e meios para o exercício dos profissionais de vigilância sanitária nos serviços de Atenção Primária à Saúde.

Este artigo propõe discutir potencialidades do GF para o desenvolvimento de pesquisa sobre Vigilância Sanitária e Atenção Primária à Saúde.

\section{Apresentação da Técnica de Grupo Focal}

Os elementos cruciais para a execução do GF são a definição e o número de participantes, o número de encontros, o local e a ambiência, a organização do espaço físico, o tempo de duração das sessões, o(s) moderador(es), o(s) observador(es), um guia ou roteiro com perguntas de relevância em relação aos objetivos do estudo, além dos dispositivos tecnológicos para registro das informações suscitadas (THOFEHRN et al., 2013).

A técnica de GF pode ser descrita de acordo com três etapas, a saber: planejamento e montagem; prática do grupo focal e análise dos dados. Para a etapa de planejamento e montagem do GF podem ser considerados os seguintes elementos: orçamento, local, ambiência, roteiro, duração, data, moderadores, observadores e, por fim, seleção dos participantes (KIND, 2004; RESSEL et al., 2008; TRAD, 2009; RODRIGUES, 2012; SOUZA et al., 2015).

O orçamento deve considerar que é necessária a provisão de verbas para a compra de aparelhos de áudio e vídeo, pagamento de profissionais para transcrição das gravações e até mesmo o custo da reserva do local para a realização do GF, caso não se disponha de espaços comunitários ou públicos. O local deve possuir características que reforcem a boa interação entre os participantes e evitem a dispersão da atenção destes. Faz parte do 
escopo destas características ser um local neutro, acessível, pouco movimentado e que possibilite a disposição dos participantes em círculo (DIAS, 2000; IERVOLINO; PELICIONI, 2001; TRAD, 2009). Os grupos focais realizados via internet permitem um ambiente mais reservado, contato privativo, que pode privilegiar o debate de temas que versam sobre tópicos constrangedores (SCHROEDER; KLERING, 2009).

Considera-se a limitação do tempo para a aplicação do roteiro no GF, por isso esse instrumento deve conter poucas questões, a fim de oportunizar a manifestação de todos. Deve ser um instrumento que flexibilize a condução do GF sem perda de tópicos relevantes que os participantes possam vir a suscitar no momento (TRAD, 2009). De acordo com alguns autores a duração do evento não deve ultrapassar duas horas, pois uma duração maior pode acarretar prejuízo dos resultados, por exaustão dos participantes (WESTPHAL et al., 1996; DEBUS, 1988; IERVOLINO; PELICIONI, 2001; MEIER; KUDLOWIEZ, 2003; ASCHIDAMINI; SAUPE, 2004). A escolha da data também é um cuidado importante para a ocorrência do evento e deve ser combinada de maneira que possa ser cumprida por todos os envolvidos (RESSEL et al., 2008).

A designação dos moderadores e observadores é feita a partir dos objetivos da pesquisa. O moderador deve se manter neutro na discussão, ser flexível, ter habilidades para conduzir a reunião, promovendo o envolvimento de todos os participantes, evitando que alguns deles monopolizem a discussão, considerando comentários relevantes e respeitando o ritmo de cada participante para possibilitar ao máximo que este se expresse espontaneamente. As colocações do moderador não devem ter caráter persuasivo nem pedagógico (DIAS, 2000; KIND, 2004). O observador deve ajudar o moderador na tarefa de condução do grupo focal, estar atento aos equipamentos de registro das informações, possuir habilidade e sensibilidade suficientes para intervir quando preciso, além de perceber e registrar em diário de campo as expressões verbais e não verbais durante a discussão e apoiar na operacionalização da montagem do grupo focal (RESSEL et al., 2008; THOFEHRN et al., 2013).

A seleção dos participantes é considerada um ponto relevante e deve estar de acordo com o tema e objetivo da pesquisa. Critérios devem ser estabelecidos como a variabilidade etária, de gênero ou de classe social e, além disso, deve-se evitar a inclusão de pessoas do mesmo círculo social ou que possuam vínculos. O recrutamento dos participantes, pode ocorrer de diversas maneiras, como por sorteio aleatório, convite por meio de líderes comunitários, por anúncio em jornais, aleatoriamente por telefone, através 
de indicações feitas por outros participantes, por atendimento de saúde ou em escolas (THOFEHRN et al., 2013; IERVOLINO; PELICIONI, 2001).

Ressalta-se que tais critérios não são uniformes e devem ser ajustados de acordo com o estudo. No caso específico de vínculos interpessoais, Maldonado, Torres e Saez (2013) pontuam como aspecto positivo para o intercâmbio de informações, durante a realização do grupo focal, a afinidade que possuíam os participantes, já que pertenciam a uma mesma comunidade e haviam sido convidados pelos seus líderes comunitários.

Para a condução do GF, recomenda-se que o moderador conduza a discussão utilizando o roteiro como instrumento base durante todo o período da discussão (THOFEHRN et al., 2013). Segundo Kind (2004), a condução do grupo pode ser dividida nas seguintes etapas: introdução/abertura, preparação, conjunto do debate, encerramento, questões posteriores à avaliação e ação posterior.

Os registros da discussão e transcrição devem ser feitos obrigatoriamente, visto que sem essa documentação, não haverá como proceder a análise dos dados. Trad (2009) recomenda pelo menos dois gravadores de áudio, visando já a fase de transcrição, no sentido de não ser perdida nenhuma informação. Destaca-se a relevância do uso de microfones, mas também aponta outros aparelhos eletrônicos, como câmeras e computadores como recursos adicionais.

Finalmente, a terceira etapa dos grupos focais é a análise dos dados, que deve ser feita pelos pesquisadores. Para tanto é necessário considerar a natureza dos dados enquanto informações subjetivas, mais especificamente, provenientes de percepções e opiniões pessoais que foram tratadas durante a interação com os outros presentes à discussão (COSTA, 2012). Em razão desta subjetividade, a análise destes dados deve ser igualmente qualitativa, sem o uso de instrumentos estatísticos (ASHIDAMINI; SAUPE, 2004).

Alguns fatores exercem influência sobre a análise dos dados, um deles diz respeito ao envolvimento dos participantes no tema em discussão, pois geralmente se trata de uma realidade vivenciada em algum contexto social do entrevistado. Em razão disso, Morgan (1988) refere que o sumário etnográfico é uma das maneiras básicas para se proceder a análise; a outra é a codificação dos dados. Em se tratando de interferência na análise do GF, são fatores intervenientes as características dos participantes, o moderador, o ambiente, o fator tempo e o conteúdo tratado.

Após a realização do GF e transcrição dos relatos os pesquisadores devem atentar para as palavras dos informantes, os seus significados, a intensidade com que foram 
proferidas e as posições tomadas, bem como o registro de ideias originais, a fim de alcançar uma análise consistente (KRUEGER, 1994).

\section{Apresentação da experiência do Grupo Focal}

Em função do objeto de estudo optou-se pela realização de dois GF: o primeiro, com técnicos da vigilância sanitária municipal e o segundo, com profissionais de saúde das equipes de saúde da família. A escolha de dois GF teve o propósito de coletar o máximo de informações possíveis de ambos os grupos, considerando que os profissionais de vigilância sanitária e das USF possuem olhares peculiares, pelo fato de ocuparem posições distintas nos serviços, por vezes contrapostas, em função de suas práticas específicas. Considera-se que a escolha contribuiu para evitar constrangimentos entre os participantes, pela possibilidade de tensões, decorrentes das posições fiscalizadores $\mathrm{x}$ fiscalizados, possibilitando um melhor aproveitamento de todos os pontos de vista.

O trabalho em vigilância sanitária também detém especificidades em relação ao trabalho em saúde em geral; em grande medida é modelado por condicionalidades inscritas nas normas jurídicas, devido à natureza regulatória e de atividade do Estado em seu exercício do poder de autoridade ou poder de polícia, que acaba por produzir certa "rejeição", devido à função fiscalizatória sobre os agentes regulados (COSTA; SOUTO, 2013). Por tais peculiaridades registra-se a potencialidade da utilização da técnica de GF por permitir a escolha dos participantes do mesmo locus de atuação, facilitando a expressão destes sobre suas práticas nos serviços de saúde.

Foram utilizados os registros em diários de campo, nos quais foram descritas as atividades programadas, dificuldades enfrentadas e estratégias utilizadas diante dos desafios postos para a realização dos GF. O trabalho foi realizado no período de fevereiro a dezembro de 2014. Durante todo o mês de maio a equipe de pesquisadores investiu esforços para o cumprimento de todas as providências elencadas para a realização do GF1, que aconteceu em 10 de junho de 2014. A partir da acumulação desta experiência a equipe executou o GF2 em 02 de setembro do mesmo ano.

Nas reuniões realizadas para a organização do GF, retomou-se o objetivo da pesquisa (SOUZA et al., 2015), no intuito de direcionar o roteiro de discussão para o GF, listando-se as necessidades para a sua operacionalização. Neste sentido, destaca-se como potencialidade do GF a utilização de um roteiro intencionalmente elaborado para atender às questões do estudo sem desconsiderar as peculiaridades dos participantes. 
Para compor o GF1 foi considerado como critério de inclusão a atuação dos profissionais nos serviços de vigilância sanitária nos Distritos Sanitários (DS), na função fiscal de controle sanitário, isto é, trabalhadores que exercem poder de polícia, a quem compete o controle sanitário dos serviços de saúde públicos e privados. Para compor o GF2 foram considerados os seguintes critérios: ser membro da Equipe de Saúde da Família (EqSF) ou da Equipe de Saúde Bucal (EqSB), compostas, respectivamente, por médico, enfermeira, auxiliar/técnico de enfermagem, odontólogo/cirurgião-dentista e auxiliar/técnico de saúde bucal, conforme a Política Nacional de Atenção Básica (BRASIL, 2017).

Cada grupo focal tem sua dinâmica própria, fato que exige dos moderadores e da equipe de pesquisa envolvida promover aos participantes um ambiente acolhedor, de modo que estes se sintam à vontade e livres para compartilhar as suas ideias e vivências com os outros integrantes. Entretanto, as atividades que aparentemente são consideradas simples podem esconder dificuldades e obstáculos, os quais foram expostos neste artigo, ao longo da descrição de suas etapas, com o intuito de apresentar a experiência vivenciada e assim contribuir com outros pesquisadores que buscam utilizar esta técnica.

Portanto, evidencia-se que o GF permite ao participante a oportunidade de diálogo que pode resultar em uma reelaboração de forma crítica sobre a temática discutida. No caso deste estudo, é possível pensar que esta nova percepção poderá ocorrer diretamente nas ações realizadas nos serviços de saúde, ambiente de trabalho dos participantes, seja no âmbito da vigilância sanitária ou da assistência.

Ressalta-se ainda a importância da técnica para a vocalização de profissionais da vigilância sanitária, área permeada de tensões pela sua natureza regulatória e certa rejeição pelos agentes submetidos aos seus princípios e normativas. Só recentemente, teve início estudos sobre estes profissionais e suas práticas também por meio de GF, revelando-se os desafios das práticas destes serviços (FERRARO et al., 2009; LEAL; TEIXEIRA, 2009; LEAL; TEIXEIRA, 2014). Esses desafios podem estar relacionados ao grande número de atividades desempenhadas por esses profissionais, em razão da ampla gama de objetos sob vigilância, interesses político-econômicos contrários (COSTA; SOUZA, 2014) e considerando, também, a característica peculiar à função de fiscal de controle sanitário que é a realização em campo de inspeções em estabelecimentos de saúde e de interesse à saúde.

$\mathrm{Na}$ experiência da pesquisa abordada neste estudo a equipe de recrutamento solicitou os nomes dos técnicos lotados na Secretaria Municipal de Saúde (SMS) e nos 
DS e os nomes dos profissionais das EqSF e EqSB das USF incluídas na pesquisa. Foram organizados sorteios, sempre realizados por duas pessoas. A experiência da equipe de estudo constituida por pesquisadores, moderadores e observadores na atividade potencializou o uso da técnica de GF no sentido de acolher e registrar os relatos para posterior análise.

A busca pelos contatos foi mais fácil com os componentes do GF1. Todavia, quanto ao GF2, essa realidade foi inversa, exigindo dos organizadores um esforço muito maior, sendo necessário recorrer a terceiros, que forneceram, inclusive, os nomes e os contatos pessoais dos gerentes das USF. No GF1 dos treze contatados, incluindo titulares e suplentes, quatro participaram, cada qual representando um DS. No GF2 estiveram presentes nove profissionais de saúde, com representação de todas as categorias das EqSF e EqSB dos diferentes DS.

O número ideal de participantes enseja divergência entre autores, com recomendações que podem variar entre seis a quinze pessoas (RESSEL et al., 2008), ou oito a dez pessoas, mas que o ideal seria de cinco a sete (DEBUS, 1988; ROSO, 1997). No entanto, todos concordam que o mais importante é que cada participante tenha a chance de interagir. De acordo com Thofehrn et al., (2013), não é recomendável GF com menos de quatro pessoas e sim entrevistas individuais. Vale ressaltar que a experiência do GF1, com apenas quatro participantes, foi enriquecedora, com espaço para reflexão aprofundada sobre a temática orientada pelo roteiro.

A equipe de pesquisadores que conduziu os GF foi formada por dois observadores e dois moderadores. A designação destes foi feita a partir dos objetivos da pesquisa, com atividades específicas e compartilhadas durante o GF. Os observadores realizaram o acolhimento dos convidados, o registro das impressões e gravação em áudio e vídeo. A participação destes foi relevante, em face, inclusive, ao imprevisto com um equipamento de gravação de vídeo que apresentou problema, obrigando a saída de parte da equipe para proceder à devida substituição. Contudo, tal fato não impossibilitou a continuidade das atividades, pois havia quatro gravadores de áudio e três de vídeos à disposição.

Aos moderadores coube a condução dos trabalhos, incluindo a abertura, a apresentação da equipe, o objetivo do encontro e as informações de cunho ético, que ensejaram a leitura do Termo de Consentimento Livre e Esclarecido (TCLE), a elucidação de dúvidas e a assinatura do documento em caso de concordância. $\mathrm{O}$ estudo foi aprovado pelo Comitê de Ética em Pesquisa do Instituto de Saúde Coletiva da UFBA, mediante 
parecer $n^{\circ}$ 364.713/2013 e submetido ao edital da Fundação de Amparo a Pesquisa do Estado da Bahia - Fapesb nº 020/2013, tendo sido aprovado com recurso financeiro.

O município, locus da pesquisa supracitada, foi uma das sedes da Copa do Mundo, e devido a esse evento de massa muitos profissionais dos serviços de vigilância sanitária estavam alocados nas ações voltadas ao evento, o que ensejou um percentual elevado de recusas para participar do GF1 pela grande quantidade de atividades no período. Outros motivos de recusa foram justificados com a alegação de insatisfação com situações relacionadas ao órgão de vigilância sanitária, por não realizarem inspeções em USF há bastante tempo e por insuficiência de pessoal.

Uma das questões que envolvem o planejamento de um GF é o orçamento. Na experiência deste grupo de trabalho o projeto de pesquisa foi financiado pela Fapesb, constando em sua previsão os custos para a realização do GF, incluindo equipamentos de áudio e vídeo e pagamento para transcrição das gravações. Cabe destacar que no caso deste estudo o uso da técnica de GF se caracterizou pelo baixo custo haja vista a disponibilidade do espaço e da equipe.

Os GF foram realizados em sala de aula de Instituição de Ensino Superior Pública, com infraestrutura necessária, conforto e ambiência favorável, fora do espaço de trabalho dos participantes, de modo a evitar possíveis constrangimentos e possibilitando a neutralidade do ambiente; essa ambiência evitou inclusive a dispersão da atenção dos participantes e possibilitou a disposição destes em círculo, fatores que potencializaram a realização da técnica (DIAS, 2000; TRAD, 2009; IERVOLINO; PELICIONI, 2001). Entretanto, a localização espacial do prédio no bairro, pode ter dificultado a pontualidade dos participantes, em função da restrição de vagas para estacionamento de veículos.

A condução do GF foi organizada com base no roteiro (THOFEHRN et al., 2013) para instruir a discussão. O roteiro utilizado foi cuidadosamente elaborado com seis perguntas sobre - condições sanitárias dos serviços e suas implicações para a saúde da população, o processo de trabalho e a gestão - levando-se em conta a limitação do tempo e a importância da garantia da manifestação de todos (TRAD, 2009) e, de modo a atender aos objetivos da pesquisa e minimizar possíveis desconfortos.

A duração da atividade do GF1 foi de 1:11:42h e do GF2 foi 2:17:32h. Comparando-se os períodos necessários para cada um, pode-se inferir que possivelmente o GF2 apresentou carga horária maior por ter uma quantidade maior de participantes, bem como pela necessidade de retomar a apresentação inicial algumas vezes, em decorrência do atraso de alguns participantes. Ressalta-se que ao final da realização dos grupos focais, 
os participantes expressaram verbalmente a satisfação em ter integrado a atividade, apesar da discussão dos autores sobre a necessidade de encerramento do grupo no máximo em duas horas, a fim de evitar prejuízo nos resultados (IERVOLINO; PELICIONI, 2001; DEBUS, 1988; WESTPHAL et al., 1996; MEIER; KUDLOWIEZ, 2003; ASCHIDAMINI; SAUPE, 2004). A data foi cuidadosamente escolhida em concordância de todos os convidados para a atividade (RESSEL et al., 2008).

Na etapa de Prática do Grupo Focal o acolhimento dos convidados contou com um lanche que foi disponibilizado aos participantes, em razão do horário em que foram realizadas as atividades. Durante a abertura dos GF1 e GF2, as moderadoras deram explicações sobre a pesquisa e seus aspectos éticos, bem como elucidaram as dúvidas. $\mathrm{O}$ encerramento da atividade se deu após o agradecimento aos participantes, os quais sugeriram a devolutiva da pesquisa em formato de atividade de extensão/cooperação técnica no cotidiano das práticas nas USF.

A condução dos GF seguiu as etapas pertinentes à técnica e utilizou instrumentos básicos, a exemplo da estratégia de acolhimento, utilização do TCLE, de roteiro, equipamentos de áudio e vídeo previamente testados (TRAD, 2009). Para a etapa da análise dos dados, após transcrição do conteúdo discutido, os GF foram analisados separadamente (KRUEGER, 1994).

Para Trad (2009), o uso desta técnica no campo da Saúde Coletiva suscita algumas limitações, como a impossibilidade de estabelecer nexos causais em razão de se tratar de uma técnica de corte transversal, a qual oferece reduzido controle de variáveis; outra limitação seria possuir amostra por conveniência. Diante disto, visando reduzir possíveis vieses dos posicionamentos dos participantes dos GF, o registro das transcrições foi triangulado com as informações obtidas a partir de outras técnicas, como a observação nas USF e com as entrevistas individuais a usuários e gestores, de modo que geraram categorias e subcategorias de análise que deram corpus ao texto de discussão dos resultados da pesquisa, os quais se constituíram objeto de análise e discussão em outros artigos (BARRETO; SOUZA; SILVA, 2016; SOUZA; BARRETO; SILVA, 2017; BARRETO; SOUZA, 2017; LIMA, et al., 2018; PAZ et al., 2016). Especificamente sobre as categorias de análise geradas a partir do grupo focal, destaca-se o artigo de Lima et al (2018), e na oportunidade do encontro dos participantes do GF, também foi produzido o estudo de Paz et al. (2016). 


\section{Conclusões}

O conhecimento e a aplicação de uma técnica de pesquisa com rigor metodológico são condições necessárias para a produção científica de qualidade; nesse sentido, este artigo descreveu as etapas para a realização de grupo focal e discutiu as estratégias e os desafios para a sua utilização potencial em estudos que envolvam sujeitos de diferentes posições, neste caso, profissionais da vigilância sanitária e da atenção primária à saúde.

Entre os desafios, destacaram-se a escolha da data para a realização dos dois grupos focais, as dificuldades no estabelecimento dos contatos com os participantes, atraso nas atividades em decorrência da estrutura do local escolhido, problemas técnicos com aparelhos eletrônicos de gravação, ainda que testados com antecedência e deficiente capacidade de comunicação com os profissionais na discussão do tema proposto. Entretanto, o planejamento de certas estratégias, como a participação de mais de um moderador, a disponibilidade de amplo material de gravação e registro, apoio técnico, a utilização de procedimentos para contato com os participantes e o estabelecimento de limites de horário para o ingresso de participantes no espaço da atividade, foram fundamentais para a superação dos problemas que emergiram no processo.

Apesar de alguns limites para sua utilização, a técnica de GF se destaca pelas suas potencialidades, valendo destacar a interação entre os participantes e a possibilidade desenvolvida no grupo de revisitar a realidade, discutida pela visão do outro, diferentemente de outras técnicas e processos de produção de dados sobre um determinado objeto de estudo.

Apesar da APS e da Estratégia Saúde da Família se caracterizarem como objetos de interesse dos pesquisadores, de forma crescente nos últimos anos, ainda são raras as pesquisas que relacionam as condições sanitárias dos serviços com a saúde da população e dos trabalhadores, ainda mais no que se refere à interação de temáticas como as de assistência e vigilância. Haja vista o estudo tratar de uma área complexa e ainda pouco estudada e que abrange atores dos âmbitos reguladores ou fiscalizadores e dos regulados ou fiscalizados, importa a discussão sobre métodos e técnicas enquanto instrumentos que podem auxiliar a vigilância sanitária e os serviços de saúde na produção do conhecimento e no desenvolvimento de pesquisas com qualidade científica. 


\section{Referências}

ASCHIDAMINI, I. M.; SAUPE, R. Grupo focal - Estratégia metodológica qualitativa: Um ensaio teórico. Cogitare enfermagem, Curitiba, v. 9, n. 1, p. 9-24, 2004

BARRETO, H. I. V.; SOUZA, M. K. B. Análise das Condições Sanitárias de Unidades de Saúde da Família com base no referencial sobre Processo de Trabalho. Revista de APS (Impresso), Juiz de Fora, v. 20, n. 4, p. 519-526, 2017.

BARRETO, L. A.; SOUZA, M. K. B; SILVA, E. A. L. Rede de atenção e condições sanitárias das unidades de saúde da família: há alguma relação? Revista Enfermagem UERJ, Rio de Janeiro, v. 24, n. 6, p. 17440, 2016.

BRASIL. Ministério da Saúde. Secretaria de Atenção à Saúde. Departamento de Atenção Básica. Política Nacional de Atenção Básica. Brasília; 2017.

COSTA, E. A.; ROZENFELD, S. Constituição da vigilância sanitária no Brasil. In: ROZENFELD, S. (org.). Fundamentos da vigilância sanitária. 1. ed. $6^{\text {a }}$ Reimpressão. Rio de Janeiro: Editora Fiocruz, 2000. p.15-40.

COSTA, E. A.; SOUTO, A. C. Área Temática de Vigilância Sanitária. In: PAIM, J. S.; ALMEIDA FILHO, N. Saúde Coletiva: Teoria e Prática. Rio de Janeiro: MEDSI, 2013, p. 327341 .

COSTA, G. S. Grupos focais: um novo olhar sobre o processo de análise das interações verbais. Revista Intercâmbio, Montes Claros, s.v., s.n., p. 153-172, 2012.

COSTA, E. A. Regulação e Vigilância Sanitária: Proteção e Defesa da Saúde. In: ROUQUAYROL, M. Z; GURGEL, M. (org.). Epidemiologia \& Saúde. 7. ed. Rio de Janeiro: Medbook, 2013. p. 493-520.

DEBUS, M. (org.). Manual para excelência em la investigación mediantegrupos focales. 1. ed. Pennsylvania: University of Pennsylvania/ Applied Communications Technology, Needham Porter Novelli, 1988.

DIAS, C. A. Grupo focal: técnica de coleta de dados em pesquisas qualitativas. Informação e Sociedade, João Pessoa, v. 10, n. 2, p. 1-12, 2000.

FERRARO, A. H. A. et al. Imagem-objetivo para a descentralização da vigilância sanitária em nível municipal. Cad Saúde Pública, Rio de Janeiro, v. 25, n. 10, p. 2201-2217, out. 2009.

IERVOLINO, A. S.; PELICIONI, M.C. F. A utilização do grupo focal como metodologia qualitativa na promoção da saúde. Revista da Escola de Enfermagem da USP, São Paulo, v. 35, n. 2, p. 115-121, 2001.

KIND, L. Notas para o trabalho com a técnica de grupos focais. Psicologia em revista, Belo Horizonte, v. 10, n. 15, p. 124-136, 2004.

KRUEGER, R. A. Focus Groups: a pratical guide for applied research. 2. ed. Thousand Oaks: SAGE Publications, 1994.

LEAL, C. O. B. S.; TEIXEIRA, C. F. Análise de situação dos recursos humanos da vigilância sanitária em Salvador - BA, Brasil. Interface. Comunicação, Saúde e Educação, Botucatu, v. 13, n. 30, p. 167-179, set. 2009. 
LEAL, C.O.B.S; TEIXEIRA, C. F. Comida de rua: um estudo crítico e multirreferencial em Salvador, BA - Brasil. Vigilância Sanitária em Debate: Sociedade, Ciência \& Tecnologia, Rio de Janeiro, v. 2, n. 4, p. 12-22, nov. 2014.

LIMA, Y. O. R. et al. Percepção dos profissionais da Vigilância Sanitária sobre as condições sanitárias dos serviços da Atenção Primária à Saúde. In: CONGRESSO IBERO-AMERICANO DE INVESTIGAÇÃ̃ QUALITATIVA EM SAÚDE, 7., 2018, Fortaleza. Atas... Fortaleza: Ludomedia, 2018. p. 386-391.

MALDONADO, V. E.M.; TORRES, L. M. T.; SAEZ, M. J. N. Grupo Focal de discusión como herramienta para el estudio de las relaciones de género en miembros de uma comunidad urbana. Revista Eletrônica Semestral de Enfermeiría, Goiânia, v. 12, n. 1, p. 436-449, 2013;

MEIER, M. J.; KUDLOWIEZ, S. Grupo focal: uma experiência singular. Texto \& Contexto Enf., Florianópolis, v. 12, n. 3, p. 394-399, 2003.

MERTON, R. K.; FISKE, M.; KENDALL, P. L. The focused interview: a manual of problems andprocedures. 2. ed. Nova York. The Free Press, 1990.

MORGAN, D. L. Focus groups as qualitative research. 2. ed. Beverly Hills: SAGE Publications, 1988.

PAZ, B. M. S. et al. Condições Sanitárias das Unidades de Saúde da Família: perspectiva dos profissionais de saúde. In: SIMPÓSIO BRASILEIRO DE VIGILÂNCIA SANITÁRIA, 7., 2016, Salvador. Anais... Rio de Janeiro: Abrasco, 2016. p. 304-304.

RESSEL, L. B. et al. O uso do grupo focal em pesquisa qualitativa. Texto \& Contexto Enf. Florianópolis, v. 17, n. 4, p. 779-786, 2008.

RODRIGUES, J. S. Infusões à base de folhas de passifloras do cerrado: compostos fenólicos, atividade antioxidante in vitro e perfil sensorial. 2012. Dissertação (Mestrado em Nutrição Humana) - Faculdade de Ciências da Saúde, Universidade de Brasília, Brasília, 2012.

ROSO, A. Grupos focais em Psicologia Social: da teoria à prática. Psico., Porto Alegre, v. 28, n. 2, p. 155-169, 1997.

SCHROEDER, C. S.; KLENRING, R. L. On-line focus group: uma possibilidade para a pesquisa qualitativa em administração. Cadernos EBAPE.BR. Rio de Janeiro, v. 7, n. 2, p. 332-348, 2009.

SOUZA, M. K. B. et al. Condições Sanitárias das Unidades de Saúde da Família: desafios para o processo de trabalho, a saúde da população e a gestão em saúde. Salvador: Instituto de Saúde Coletiva. Universidade Federal da Bahia; 2015. No prelo.

SOUZA, M. K. B.; BARRETO, L. A.; SILVA, E. A. L. Análise de conformidade e não conformidade de unidades de saúde da família. Revista Baiana de Enfermagem, Salvador, v. 31, n. 4, p. e18247, 2017.

THOFEHRN, M. B. et al. Grupo focal: una técnica de recogida de datos em investigaciones cualitativas. Index de enfermeira, Rio de Janeiro, v. 22, n. 1-2, p. 75-78, jun. 2013.

TRAD, L. A. B. Grupos focais: conceitos, procedimentos e reflexões baseadas em experiências com o uso da técnica em pesquisas de saúde. Physis Revista de Saúde Coletiva, Rio de Janeiro, v.19, n. 3, p. 777-796, 2009. 
WESTPHAL, M. F. et al. Grupos focais: experiências precursoras em programas educativos em saúde no Brasil. Bol. Oficina Sanit. Panam, Washington, v. 120, n. 6, p. 472-481, jun. 1996.

Recebido em: 22 de dezembro de 2017.

Aceito em: 08 de abril de 2019. 